\title{
Special issue: supermodularity and monotone methods in economics
}

\author{
Rabah Amir ${ }^{1}$ \\ Published online: 25 September 2018 \\ ๑) Springer-Verlag GmbH Germany, part of Springer Nature 2018
}

\begin{abstract}
This special issue brings together eight separate contributions reflecting recent advances in the methodology of supermodular optimization/games and monotone methods. Two of the papers extend some aspects of the methodology itself: Barthel and Sabarwal (Econ Theory, 2018. https://doi.org/10.1007/s00199-017-1079-3) deal with comparative statics without the strong set order on constraints and Beggs (Econ Theory, 2018. https://doi.org/10.1007/s00199-016-0987-y) deals with the case of boundary optimal solutions. The next five papers deal with various economic applications, which may be classified into two broad groups. The first of these deals with dynamic general equilibrium within macroeconomic dynamics (Datta et al. in Econ Theory, 2018. https://doi.org/10.1007/s00199-017-1046-z; Barbie and Hillebrand in Econ Theory, 2018. https://doi.org/10.1007/s00199-017-1082-8). The second group of papers consists of studies applying the techniques at hand to industrial organization (Reynolds and Rietzke in Econ Theory 2018. https://doi.org/10.1007/s00199-016-0963-6; Cosandier et al. in Econ Theory, 2018. https://doi.org/10.1007/s00199-017-1050-3) and applied microeconomic theory (Dekel and Pauzner in Econ Theory, 2018. https://doi.org/ 10.1007/s00199-017-1083-7). Finally, the paper by Bich et al. (Econ Theory, 2018. https://doi.org/10.1007/s00199-017-1045-0) considers an alternative general formulation of a theoretical framework for dynamic programming.
\end{abstract}

Keywords Complementarity · Dynamic general equilibrium · Supermodular games · Submodular games · Monotone comparative statics

JEL Classification D51 $\cdot$ E13 $\cdot$ C61 $\cdot$ C62 $\cdot$ C72 $\cdot$ L13 $\cdot$ L51

It is a pleasure to acknowledge helpful conversations and useful feedback on the multiple topics of this special issue with Manjira Datta, Jacques Dreze, Kevin Reffett, Tarun Sabarwal, Xavier Vives and Lukasz Wozny.

$凶$ Rabah Amir

rabah-amir@uiowa.edu

1 Department of Economics, University of Iowa, Iowa City, IA 52242, USA 


\section{Introduction}

Supermodular optimization is a new methodology for conducting comparative statics or sensitivity analysis, or determining how changes in exogenous parameters affect endogenous variables in optimization models. This methodology is often referred to as lattice programming, since the most general formulation postulates that the parameter space is a partially ordered set and the action set is a lattice. Originally developed within the fields of operations research and mathematical programming by Topkis (1968, 1978) and Veinott (1989), this methodology has found its way into economics, as a major methodological breakthrough that now forms the very basis of modern monotone comparative statics and of the theory of supermodular games. Early studies in economics include Vives (1990) and Milgrom and Roberts (1990) for the general theory, and a number of applications (e.g, Amir et al. 1991 for comparative dynamics, and others). ${ }^{1}$

The use of this methodology now permeates several different strands of literature in economic theory, and the conclusions therefore derived are often one of the main motivations behind the construction of a model. These conclusions also often form the theoretical grounding behind much work on empirical testing. The main characteristic of this methodology is that it relies essentially on critical assumptions for the desired monotonicity conclusions and dispenses with superfluous assumptions that are often imposed only to facilitate the use of the classical method, which is based on the implicit function theorem and requires smoothness, interiority and concavity conditions. The main insight is indeed quite simple. If, in a maximization problem, the objective function reflects a complementarity between an endogenous variable and an exogenous parameter, in the sense that having more of one increases the marginal returns to having more of the other, then the optimal value of the former will be increasing in the latter. In the case of multiple endogenous variables, then all of them must also be complements in order to guarantee that their increases are mutually reinforcing. This conclusion follows directly from the underlying complementarity relationship and is thus independent of the aforementioned superfluous assumptions. It thus holds even if there are multiple optimal values of the endogenous variables.

Another major advance due to this framework of analysis is the theory of supermodular games, better known in economics as games with strategic complementarities, introduced by Topkis (1979). The main characteristic of these games is that they possess increasing reaction correspondences (that need not be continuous), reflecting a complementarity relationship between own actions and rivals' actions. As a consequence of Tarski's fixed-point theorem, the latter property guarantees the existence of pure-strategy Nash equilibrium points. Because the latter type of equilibrium is most often the desired concept in economic models, the scope of game-theoretical modeling in economics is therefore substantially enlarged. Another key observation in this respect is that supermodularity is again the relevant notion behind the monotone comparative statics of Nash equilibrium points. Furthermore, it will be argued that supermodular games are more conducive to predictable comparative statics properties

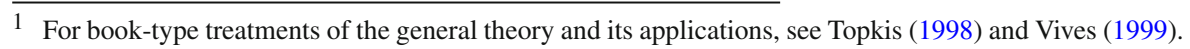


than games with continuous best responses, the latter being the other class of games that always admit pure-strategy Nash equilibrium points.

One might ask whether a new look at complementarity and its attending methodology was needed. Topkis (1998, p. 3) quotes Samuelson (1947) as asserting that: "In my opinion the problem of complementarity has received more attention than is merited by its intrinsic importance." Yet much later Samuelson (1974) had an ominous change of mind and wrote that "The time is ripe for a fresh modern look at the concept of complementarity. The last word has not yet been said on this ancient preoccupation of literary and mathematical economists. The simplest things are often the most complicated to understand fully."

This special issue brings together eight separate contributions reflecting recent advances in the methodology of supermodular optimization/games and monotone methods. Two of the papers extend some aspects of the methodology itself: Barthel and Sabarwal (2018) deal with comparative statics without the strong set order on constraints and Beggs (2018) deals with boundary solutions of parametric optimization and of equilibrium problems. The remaining six papers consider various economic applications, which may be classified into two broad groups. The first of these deals with dynamic general equilibrium and overlapping generations models within macroeconomic dynamics (Datta et al. 2018; Barbie and Hillebrand 2018). The second group of papers consists of studies in industrial organization (Cosandier et al. 2018; Reynolds and Rietzke 2018) and applied microeconomic theory (Dekel and Pauzner 2018). Of the latter three papers, the first is a game of strategic complements, while the other two are games of strategic substitutes (under general assumptions on the primitives of the three games). Finally, the paper by Bich et al. (2018) considers an alternative general formulation of a new theoretical framework for dynamic programming.

In what follows, we shall refer to monotone comparative statics problems in the following form: Let $X$ be a lattice, $A, B$ are subsets of $X$ ordered by some relation $\sqsubseteq$, and $f: X \times T \rightarrow R$, where $T$ is a partially ordered set. When is it true that $A \sqsubseteq B$ and $t \preceq t^{\prime} \Rightarrow \arg \max _{A} f(\cdot, t) \sqsubseteq \arg \max _{B} f\left(\cdot, t^{\prime}\right)$ ? In other words, when is $\arg \max _{A} f(\cdot, t)$ increasing in $(A, t)$ ? This is the form used by Milgrom and Shannon (1994) whereas Topkis's $(1968,1978)$ original formulation kept the parametrized constraint set fixed, and considered monotonicity of the optimal solutions only w.r.t. the parameter $t$.

\section{Directional monotone comparative statics}

In order to describe the paper by Barthel and Sabarwal (2018), we begin with some background. The Topkis-Milgrom-Shannon theorem proves that when $\sqsubseteq$ is the strong lattice set order, $\arg \max _{A} f(\cdot, t)$ is increasing in $(A, t)$ in the strong set order if and only if for every $t \in T, f(\cdot, t)$ is quasi-supermodular on $X$ and $f$ satisfies the singlecrossing property on $X \times T$. A well-known limitation of Milgrom and Shannon (1994) is that it does not apply to some basic economic problems in which constraint sets are not ordered in the strong set order. A classical example is the standard Walrasian budget set parameterized by wealth. Quah (2007) introduces new machinery to work with budget-type constraints in Euclidean spaces and to determine when optimizers 
are (weakly) increasing in particular directions. His techniques include new binary relations, a new set order, termed $\mathcal{C}_{i}$-flexible set order, and a new notion of $\mathcal{C}_{i}$-quasisupermodular function. This new machinery has proved very insightful, but harder for a broader audience to apply to actual models.

Barthel and Sabarwal (2018) extends the monotonicity theorem of Milgrom and Shannon (1994) to Quah-ordered constraint sets and characterizes monotone comparative statics in different directions in finite-dimensional Euclidean spaces. Their work is notable for showing more clearly the connection between the two earlier papers. A theoretical benefit of their approach is the development of results that retain the more transparent flavor of their counterparts in the earlier theory. In addition, starting from Quah's sufficient conditions, the characterization given here has a natural formulation in terms of cardinal assumptions: i-supermodular and i-increasing differences, which, in turn, have a new and natural formulation in terms of easily verifiable differential conditions using directional derivatives. In addition to being part of the development of new tools to apply the monotone comparative statics results at hand, these results also help ground the entire approach more soundly in the standard theory.

A strong aspect of this paper is manifested in the scope for additional economic applications that can be analyzed with their approach, which include in particular game-theoretical settings, made possible by the fact that their framework encompasses parameterized objective functions (absent in Quah 2007), a necessary feature for gametheoretical settings. The authors extend the key application of normality of demand in basic consumer theory, already provided by Quah (2007), to the case of parameterized utility functions (as in the case of Geary-Stone utilities) or of a limited set of discrete goods. They also provide illustrations of their results to the following models: A form of multi-market competition by firms, the analysis of a polluting firm choosing abatement levels and outputs, and the analysis of multi-unit auctions with an aggregate budget constraint for each bidder. Along with Quah (2007), this work ushers new hope for opening additional avenues for broader use of these important tools in economic applications.

\section{Markov equilibria of dynamic general equilibrium models}

This special issue contains two papers dedicated to various applications of lattice programming and monotone methods to macroeconomic dynamic general equilibrium theory.

Datta et al. (2018) investigates recursive equilibrium in economies with complementarities propose a new method to characterize minimal state-space recursive equilibrium for a broad class of infinite horizon dynamic general equilibrium models. This class includes models for which there are no known previous existence results, as well as model for which equilibrium has been claimed to be indeterminate (see, e.g., Santos 2002). Their new approach is based on results from powerful fixed-point theory for monotone operators defined on an equilibrium version of the household's Euler equation, with in addition the equilibrium operator being defined in two distinct steps. The methodology thus amounts to a significant extension of the method used in previous contributions by Coleman (1991) (also see Datta et al. 2002; Mirman et al. 
2008, among others). Intuitively, in the first step, the authors construct solutions to a parameterized fixed-point problem that guarantee necessary structural restrictions implied by household optimization relative to individual state variables. Then, using a fixed-point monotone comparative statics result on first step fixed points, the authors define a second-step monotone operator, which satisfies the necessary aggregate state consistency conditions for a recursive equilibrium. This is a key methodological contribution toward the development of appropriate tools to study recursive equilibrium in a broad class of economies with sufficient static and dynamic complementarities. Moreover, to circumvent the problem of equilibrium indeterminacy in such classes of models, the authors' partial ordering methods also provide a theory of multiple equilibrium comparative statics, thus complementing for instance recent results obtained by Acemoglu and Jensen's (2015). In contrast to the literature on local indeterminacies (using the methods of smooth dynamical systems to characterize sequential equilibrium near steady states), the present equilibrium comparison results are shown to be computable globally via successive approximations from appropriate extremal elements in sets of candidate equilibrium function spaces.

At this point, it might be instructive to point out that the term "monotone methods" may be taken in its broadest sense as encompassing the theory of supermodular optimization and games, among other aspects. However, the most frequent use of this phrase actually occurs in the study of economic dynamics and refers more specifically to the use of lattice programming techniques, along with versions of the TarskiKantorovich-Katsner fixed-point theorem (Tarski 1955), and of the study of a self-map on consumption functions constructed directly from Euler equations in economic dynamics (as in Lucas and Stokey 1987; Coleman 1991; Datta et al. 2018; Barbie and Hillebrand 2018, among others). To deal with the uniqueness issue, the study of this mapping also sometimes involves another lesser-known fixed-point argument for concave maps due to Krasnosel'skii and Zabreiko (1984). ${ }^{2}$

Barbie and Hillebrand (2018) considers a general class of frictionless overlapping generations economies with capital accumulation and stochastic production shocks. The underlying class of dynamic economies postulates the usual general set up including time-additive (discounted) consumer utility and independent and identically distributed TFP shocks. The main focus of the paper is on the existence and general properties of bubbly Markov equilibria (defined as recursive equilibria, with a Markov stationary structure with respect to a natural state space, which admit a nontrivial bubble). As in prior work on dynamic economies by Coleman (1991), Datta et al. (2002) and Morand and Reffett (2007), the present authors invoke monotone methods to develop a general approach to construct Markov equilibria and provide necessary and sufficient conditions for these equilibria to be bubbly. This powerful methodology is thus adapted to account for the presence of bubbles here. In addition, this method is of a constructive nature and can thus be directly employed to compute bubbly Markov equilibria numerically in applications of our results. The main result of the paper shows that a bubbly Markov equilibrium exists whenever the bubbleless equilibrium is Pareto inefficient (according to the concept of interim or conditional

2 Pioneering work on lattice programming in economic dynamics includes Amir et al. (1991), Hopenhayn and Prescott (1992), and Amir (1996b) in single-agent settings, as well as Amir (1996c) and Curtat (1996) for dynamic (stochastic) games. For more recent work, see Balbus et al. (2018) and references therein. 
sense) due to either over-accumulation of capital or to inefficient risk sharing between generations. In such cases, a bubbly asset can be welfare improving by permitting statecontingent transfers between generations, which otherwise would not be possible. The presence of bubbles and the main result of the paper underscore key differences with the deterministic case (see, e.g., Tirole 1985; Aiyagari and Peled 1991), which the authors argue motivates the need for the monotone methods used here, in conjunction with some other tools from the literature on overlapping generations economies.

\section{Games of strategic complements and substitutes}

This special issue offers three papers concerned with various applications of the theory of games of strategic complements and substitutes, and to the study of the comparative statics properties of their pure-strategy Nash equilibrium points.

Cosandier et al. (2018) considers a Bertrand duopoly with differentiated products, wherein an exogenous proportion of consumers (defined as a measure of market transparency) are aware of the existence of only one firm (and thus only one product). While these unaware consumers are captive to the one firm they know of, the rest of the consumers may patronize both firms, as in the standard model. The main purpose of the paper is to investigate the effects of such transparency on prices and market performance. Existence of Bertrand equilibrium is established by making minimal assumptions to fit the basic results from the theory of supermodular games. The authors first consider the case where prices are strategic complements, the typical configuration for Bertrand competition, and show that the conventional wisdom concerning prices is confirmed, in that they decrease with market transparency. Using insights from supermodular games, this analysis is conducted at a high level of generality. In addition, consumer surplus always increases with higher transparency, but changes in firms' profits are ambiguous. On the other hand, for the (somewhat uncommon) case of prices as strategic substitutes, an increase in market transparency may lead to an increase in (at most) one of the prices, which may generate ambiguous effects on both consumer surplus and firms' profits. To obtain a more complete set of results, in a final section the authors resort to the widely used setting of a duopoly with linear demand for differentiated products and obtain closed-form solutions that yield a more complete picture of the effects of higher transparency. The results of the paper provide one particular theoretical perspective on the mixed evidence concerning the effects of the Internet (as a proxy for higher transparency) on retail prices. This angle is distinct from, though closely related to, the more common search-theoretical approaches to the same issue. ${ }^{3}$

Reynolds and Rietzke (2018) reconsider the classical issue of price caps in regulation theory for oligopoly markets in the presence of endogenous entry (with sunk entry costs). Existing studies on this topic have established that, for Cournot oligopoly with deterministic demand and constant marginal cost, exogenously reducing a price cap increases total output, consumer welfare, and total welfare, outcomes that are consistent with those for oligopoly markets with a fixed number of firms (see e.g.,

\footnotetext{
${ }^{3}$ For recent work on price competition and supermodularity, see Prokopovych and Yannelis (2018).
} 
Amir and Lambson 2000). With deterministic demand and increasing marginal cost, these comparative statics results may be fully reversed, and a welfare-improving cap may not exist. Recent results in the literature show that, with a fixed number of firms, stochastic demand and constant marginal cost, lowering a price cap may either increase or decrease output and welfare (locally); however, a welfare-improving price cap does exist in a suitable price interval. In contrast to these recent results, Reynolds and Rietzke show that a welfare-improving cap may not exist if entry is endogenous. However, within this stochastic demand environment, the authors identify simple, general and economically meaningful conditions on the curvature of demand that are sufficient to ensure the existence of a welfare-improving cap when entry is endogenous. The two key conditions on demand are that it is supermodular in output and the (random) demand shifter and that the monopolist's marginal revenue is decreasing in output. The latter condition is due to Novshek (1985) and guarantees that a firm's profit is submodular in own and rivals' outputs, so that its reaction curve is downward sloping in rivals' output (for more on this point, see Amir 1996a; Ewerhart 2014). The latter property, which is equivalent to the presence of the business-stealing effect in Cournot oligopoly, is then argued to be crucially needed for the existence of a welfare-enhancing price cap.

Dekel and Pauzner (2018) considers a class of two-player, two-action (high, $H$, and low, $L$ ) symmetric games of incomplete information. In the $2 \times 2$ matrix, with the two payoffs to $L$ normalized to zero, the payoffs are submodular for any given type (i.e., $H$ does better against $L$ than against $H$ ). With threshold Bayesian strategies, the game inherits the submodularity of the matrix game (in the two thresholds as actions). The authors provide respective sufficient conditions under which there is either a unique (pure Bayesian-Nash) equilibrium which is stable (in the sense of best-reply dynamics) and symmetric, or a unique pair of mirror-image asymmetric equilibria that are stable (together with an unstable symmetric equilibrium). In other words, (i) there is always a unique stable equilibrium, (ii) it is either symmetric or asymmetric, and hence, (iii) a very simple local condition-stability of the symmetric equilibrium (i.e., the slope of the best-response function at the symmetric equilibrium)-identifies which case applies. Stepping back, the authors provide simple respective sufficient condition on primitives (the distribution function of players' type, and the two payoff constants corresponding to action $H$ ) for when the unique stable equilibrium is asymmetric and symmetric. Despite the well-known lack of general results on the comparative statics of games of strategic substitutes, the authors show that the conditions guaranteeing the uniqueness described above also yield novel comparative statics results for this class of games. ${ }^{4}$

Last but not least, the authors demonstrate that this Bayesian game setting has a broad scope of possible applications in applied microeconomics, by illustrating its suitability in three different models: A model of public good provision where the cost of provision is private information, a model of R\&D or capacity expansion, and a novel model of gender differences in career choices. This paper joins a growing literature dedicated to symmetry breaking and the emergence of inter-player heterogeneity (or

\footnotetext{
4 For more on the issue of comparative statics for submodular games, see Milgrom and Roberts (1990), Roy and Sabarwal (2012), Echenique (2002), Acemoglu and Jensen (2009), Monaco and Sabarwal (2016), and Amir et al. (2010).
} 
endogenous diversity) in various strategic settings: see Matsuyama (2002), Amir et al. (2010) and Acemoglu et al. (2017).

\section{Comparative statics of boundary solutions}

As the single paper on this original topic in this special issue, Beggs (2018) studies the sensitivity of economic equilibria to perturbations when the implicit function theorem cannot be applied on account of the presence of boundaries, in particular in the commonly encountered form of linear nonnegativity constraints in economic analysis. It presents results from the mathematical programming literature (see specific references in Beggs 2018) dealing with variational inequalities, which provide conditions under which equilibria are robust to perturbation and are locally unique Lipschitz continuous functions of parameters (as smoothness does not generally hold in these situations). The main issue here arises for the implicit function theorem when the set of constraints which bind may change if agents' payoff functions are perturbed. The generalization of the implicit function theorem given requires that a certain family of determinants be nonzero and has the same sign regardless of which constraints one treats as binding. More geometrically, the determinant of the Jacobian matrix is required to have the same nonzero sign when restricted to certain subspaces.

The examples given show that how these conditions tend to be easily verifiable. In particular, in the examples of search equilibrium and Cournot, they will hold, if own effects are strong enough. Equivalent geometrical conditions are also given. The author presents some selected economic applications including search equilibrium, Cournot equilibrium and general equilibrium to illustrate the use of the results here on equilibrium-type problems, since the optimization case was already covered in the economics literature.

While dealing explicitly with an important issue arising in common monotone comparative statics exercises in economics, this paper deals with settings in which the solutions are strongly continuous in the parameters in the spirit of the classical implicit function theorem.

\section{Generalized dynamic programming}

As the single paper on the foundation of dynamic programming topic in this special issue, Bich et al. (2018) develop a generalized dynamic programming approach for evaluation criteria based on aggregating reward functions that depend on the current action and the future expected payoff. Some minimal regularity assumptions, which may be viewed in the same spirit as axioms, are proposed on the aggregator to establish existence, uniqueness and computation of the solution to the Bellman equation.

The proposed setting allows to encompass and generalize many previous results based upon additive or nonadditive one-period reward functions, including the standard (exponentially) discounted model, the recursive preference model by Koopmans, the bi-convergence approach by Streufert, and the so-called Blackwell and Thompson aggregators. While recent work with a similar motivation by Kamihigashi (2014) used 
a lattice-theoretical approach centered around Tarski's fixed-point theorem (instead of the standard Banach contraction fixed-point theorem), Bich, Drugeon and Morhaim's approach relies instead on a novel topological approach that exploits a weak continuity assumption on the aggregator.

Finally, the authors also provide a computational algorithm that converges to the value function starting from a suitable initial guess function, which is clearly a very desirable property for such a basic theory of dynamic programming, thereby making their approach constructive. For a comparison of the present results with those obtained via a lattice-theoretical methodology, see Kamihigashi (2014).

\section{References}

Acemoglu, D., Jensen, M.K.: Aggregate comparative statics. Games Econ. Behav. 81, 27-49 (2009)

Acemoglu, D., Jensen, M.: Robust comparative statics in large dynamic economies. J. Polit. Econ. 123, 587-640 (2015)

Acemoglu, D., Robinson, J., Verdier, T.: Asymmetric growth and institutions in an interdependent world. J. Polit. Econ. 125, 1245-1305 (2017)

Aiyagari, R., Peled, D.: Dominant root characterization of Pareto optimality and the existence of optimal equilibria in stochastic overlapping generations models. J. Econ. Theory 54, 69-83 (1991)

Amir, R.: Cournot oligopoly and the theory of supermodular games. Games Econ. Behav. 15, 132-148 (1996a)

Amir, R.: Sensitivity analysis in multisector optimal economic dynamics. J. Math. Econ. 25, 123-141 (1996b)

Amir, R.: Continuous stochastic games of capital accumulation with convex transitions. Games Econ. Behav. 15, 111-131 (1996c)

Amir, R., Garcia, F., Knauff, M.: Symmetry-breaking in two-player games via strategic substitutes and diagonal nonconcavity: a synthesis. J. Econ. Theory 145, 1968-1986 (2010)

Amir, R., Lambson, V.: On the effects of entry in Cournot markets. Rev. Econ. Stud. 67, 235-254 (2000)

Amir, R., Mirman, L., Perkins, W.: One-sector nonclassical optimal growth: optimality conditions and comparative dynamics. Int. Econ. Rev. 32, 625-644 (1991)

Balbus, Ł., Dziewulski, P., Reffett, K., Wozny, Ł.: A qualitative theory of large games with strategic complementarities. Econ. Theory (2018). https://doi.org/10.1007/s00199-017-1075-7

Barbie, M., Hillebrand, M.: Bubbly Markov equilibria. Econ. Theory (2018). https://doi.org/10.1007/ s00199-017-1082-8

Barthel, A.-C., Sabarwal, T.: Directional monotone comparative statics. Econ. Theory (2018). https://doi. org/10.1007/s00199-017-1079-3

Beggs, A.: Sensitivity analysis of boundary equilibria. Econ. Theory (2018). https://doi.org/10.1007/ s00199-016-0987-y

Bich, P., Drugeon, J.-P., Morhaim, L.: On temporal aggregators and dynamic programming. Econ. Theory (2018). https://doi.org/10.1007/s00199-017-1045-0

Coleman II, W.J.: Equilibrium in a production economy with an income tax. Econometrica 59, 1091-1104 (1991)

Cosandier, C., Garcia, F., Knauff, M.: Price competition with differentiated goods and incomplete product awareness. Econ. Theory (2018). https://doi.org/10.1007/s00199-017-1050-3

Curtat, L.: Markov equilibria in stochastic games with complementarities. Games and Economic Behavior 17, 177-199 (1996)

Datta, M., Mirman, L., Reffett, K.: Existence and uniqueness of equilibrium in distorted dynamic economies with capital and labor. J. Econ. Theory 103, 377-410 (2002)

Datta, M., Reffett, K., Woźny, Ł.: Comparing recursive equilibrium in economies with dynamic complementarities and indeterminacy. Econ. Theory (2018). https://doi.org/10.1007/s00199-017-1046-z

Dekel, E., Pauzner, A.: Uniqueness, stability and comparative statics for two-person Bayesian games with strategic substitutes. Econ. Theory (2018). https://doi.org/10.1007/s00199-017-1083-7 
Echenique, F.: Comparative statics by adaptive dynamics and the correspondence principle. Econometrica 70, 833-844 (2002)

Ewerhart, C.: Cournot games with bi-concave demand. Games Econ. Behav. 85, 37-47 (2014)

Hopenhayn, H.A., Prescott, E.C.: Stochastic monotonicity and stationary distributions for dynamic economies. Econometrica 60, 1387-1406 (1992)

Kamihigashi, T.: Elementary results on solutions to the Bellman equation of dynamic programming: existence, uniqueness, and convergence. Econ. Theory 56, 251-273 (2014). https://doi.org/10.1007/ s00199-013-0789-4

Krasnosel'skii, M.A., Zabreiko, P.P.: Geometrical Methods of Nonlinear Analysis. Springer, Berlin (1984)

Lucas Jr., R.E., Stokey, N.L.: Money and interest in a cash-in-advance economy. Econometrica 55, 491-513 (1987)

Matsuyama, K.: Explaining diversity: symmetry-breaking in complementarity games. Am. Econ. Rev. 92, 241-246 (2002)

Milgrom, P., Roberts, J.: Rationalizability, learning, and equilibrium in games with strategic complementarities. Econometrica 58, 1255-1278 (1990)

Milgrom, P., Shannon, C.: Monotone comparative statics. Econometrica 62, 157-180 (1994)

Mirman, L., Morand, O., Reffett, K.: A qualitative approach to Markovian equilibrium in infinite horizon economies with capital. J. Econ. Theory 139, 75-98 (2008)

Monaco, A.J., Sabarwal, T.: Games with strategic complements and substitutes. Econ. Theory 62, 65-91 (2016). https://doi.org/10.1007/s00199-015-0864-0

Morand, O., Reffett, K.: Stationary Markovian equilibrium in overlapping generations models with stochastic nonclassical production and Markov shocks. J. Math. Econ. 43, 501-522 (2007)

Novshek, W.: On the existence of Cournot equilibrium. Rev. Econ. Stud. L II, 85-98 (1985)

Prokopovych, P., Yannelis, N.: On strategic complementarities in discontinuous games with totally ordered strategies. J. Math. Econ. 70, 147-153 (2018)

Quah, J.K.-H.: The comparative statics of constrained optimization problems. Econometrica 75, 401-431 (2007)

Reynolds, S., Rietzke, D.: Price caps, oligopoly, and entry. Econ. Theory (2018). https://doi.org/10.1007/ s00199-016-0963-6

Roy, S., Sabarwal, T.: Characterizing stability properties in games with strategic substitutes. Games Econ. Behav. 75, 337-353 (2012)

Samuelson, P.: Foundations of Economic Analysis, Harvard Economic Studies, vol. 80. Harvard University Press, Cambridge (1947)

Samuelson, P.: Complementarity. J. Econ. Lit. 12, 1255-1289 (1974)

Santos, M.: On non-existence of Markov equilibria in competitive-market economies. J. Econ. Theory 105, 73-98 (2002)

Tarski, A.: A lattice-theoretic fixed point theorem and its applications. Pac. J. Math. 5, 285-309 (1955)

Tirole, J.: Asset bubbles and overlapping generations. Econometrica 53(6), 1499-1528 (1985)

Topkis, D.: Ordered optimal solutions. Ph.D. Dissertation, Stanford University (1968)

Topkis, D.: Minimizing a submodular function on a lattice. Oper. Res. 26, 305-321 (1978)

Topkis, D.: Equilibrium points in nonzero-sum $n$-person submodular games. SIAM J. Control Optim. 17, 773-787 (1979)

Topkis, D.: Submodularity and Complementarity. Princeton University Press, Princeton (1998)

Veinott, A.: Lattice programming. Unpublished notes from lectures delivered at John Hopkins University (1989)

Vives, X.: Nash equilibrium with strategic complementarities. J. Math. Econ. 19, 305-321 (1990)

Vives, X.: Oligopoly Pricing: Old Ideas and New Tools. MIT Press, Cambridge (1999)

Vives, X.: Complementarities and games: new developments. J. Econ. Lit. 43, 437-479 (2005) 